\title{
Two-Phase Resonant Converter to Drive High-Power LED Lamps
}

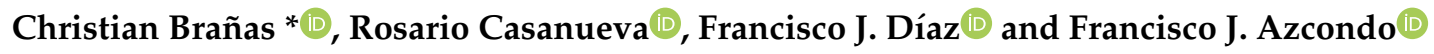 \\ Electronics Technology, Systems and Automation Engineering Department, University of Cantabria, Ave. de los \\ Castros 46, 39005 Santander, Spain; casanuer@unican.es (R.C.); diazrf@unican.es (F.J.D.); \\ azcondof@unican.es (F.J.A.) \\ * Correspondence: branasc@unican.es
}

Received: 29 November 2019; Accepted: 27 December 2019; Published: 30 December 2019

\begin{abstract}
This paper presents the design and modeling of a two-phase resonant converter that drives a LED lamp with a high-frequency pulsed current free of instabilities and flicker effect, fulfilling the recommendations of the IEEE PAR 1789-2015, so that it enables visible light-based communication at a $10 \mathrm{kB} / \mathrm{s}$ bit rate. The dynamic study of the converter takes into consideration the effect of the reflected impedance of the output filter on the AC side. In order to evaluate the dynamic response of the converter, a Spice model is defined. A $120 \mathrm{~W}$ prototype intended for street lighting applications has been built to validate the analysis and modeling.
\end{abstract}

Keywords: light sources; LED lamps; lighting control; small-signal modeling; closed-loop systems; phase shifters

\section{Introduction}

Solid state lighting (SSL) is the most recent revolution in the lighting field [1], which paves the way to developing new capabilities such as a precise wide-range dimming and visible light communication (VLC), compatible with the illumination service. High-performance dimming is highly desirable to achieve energy savings. LEDs' operation at their nominal current prevents the degradation of light quality parameters [2]. For this reason, dimming or turning on/off the nominal current level through the LEDs, according to a PWM pattern, modifying the average current, is preferred over the current amplitude modulation. A fast response of the LED current controller is required to achieve PWM dimming operation free of any flicker perception. Recently, the IEEE PAR1789 recommended a minimum frequency of $1250 \mathrm{~Hz}$ to implement PWM dimming [3]. Moreover, a fast dynamic response of the LED driver also enables the communication by light pulses, which is an interesting feature for positioning autonomous vehicles, home automation solutions, and management of lighting systems [4,5], among other applications [6]. This paper focuses on the analysis of the dynamic response of a phase-controlled series-parallel resonant converter, designed as a current source, for LED lamp driver applications. Different models have been proposed to study the dynamic response [7] of resonant converters. The small-signal modeling of resonant converters is a complex task, taking into account the number of reactive elements and operation modes. This work proposes a reduced-order model of the converter, which is valid at the bandwidth of interest. The model is defined in the LT-Spice environment providing technical insight and better understanding of the circuit. The effect of the reflected impedance of the output filter on the AC side is analyzed and then included in the model.

The paper is organized as follows: Section 2 presents the inverter and rectifier sections of the two-phase series-parallel resonant converter, deriving an equivalent circuit and the phase modulation principle using the fundamental approximation. In Section 3, the reduced small-signal model is 
obtained from the envelope model of the converter. The experimental validation of the model is presented in Section 4, finalizing with the discussion of the results.

\section{Two-Phase Series-Parallel Resonant Converter}

The two-phase series-parallel $\left(L C_{s} C_{p}\right)$ resonant converter [8], shown in Figure 1, consists of the resonant inverter stage loaded by a center tap rectifier [9].

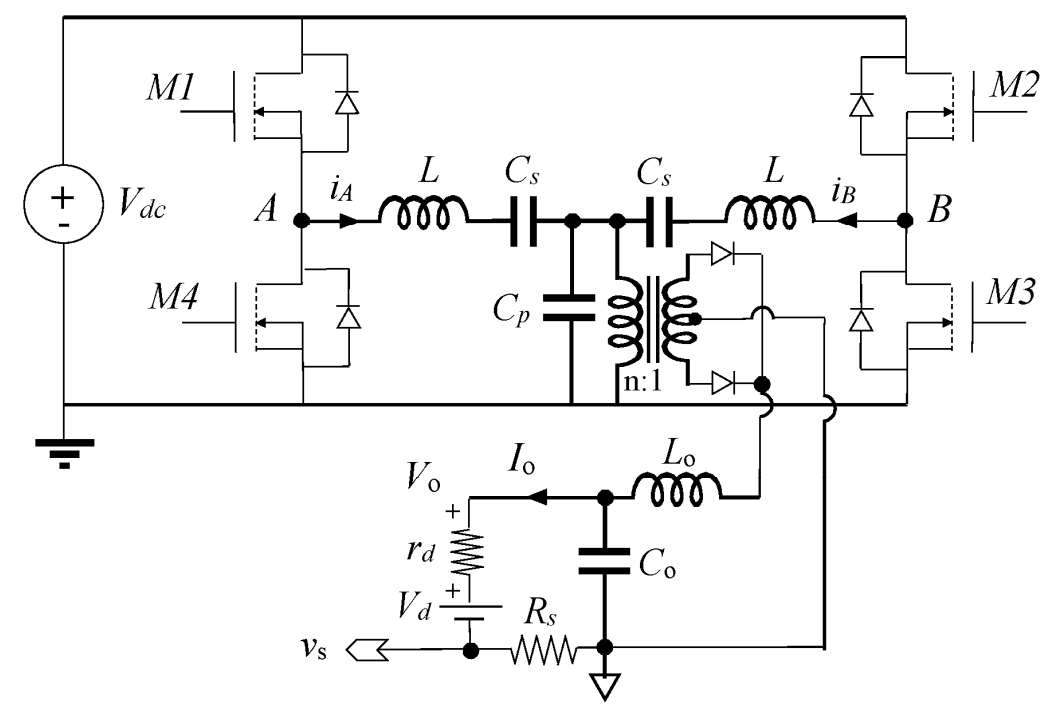

Figure 1. Two-phase $L C_{s} C_{p}$ resonant converter.

The transformer with turns-ratio $n: 1$ provides galvanic isolation. The shunt resistor $R_{S}$ is the sensor of the lamp current. The LED lamp is modeled with the dynamic resistance, $r_{d}$, and the junction voltage drop $V_{d}$, i.e., a linear model of the diode.

\subsection{The $L C_{s} C_{p}$ Resonant Inverter Stage}

In steady state, the center tap rectifier is first modeled by an equivalent resistance $R_{a c}$ on the AC side. The Fundamental Harmonic Approximation (FHA) [9] is then applied to obtain a large signal analysis of the circuit.

The midpoint voltages $v_{a}$ and $v_{b}$ in Figure 2 are represented in the complex domain by their exponential forms,

$$
\mathbf{V}_{A, B}=\frac{2 V_{d c}}{\pi} \cdot e^{ \pm j(\Psi / 2)}
$$

where $\Psi$ is the phase displacement between $v_{a}$ and $v_{b}$. The circuit in Figure 2 exhibits a symmetrical structure, where the ground-connected load can be decomposed into two paralleled halves, $2 R_{a c}$, along the symmetry axis [8]. Decomposing $\mathbf{V}_{A}$ and $\mathbf{V}_{B}$ into their orthogonal components, it is observed that the imaginary parts have equal amplitude and $180^{\circ}$ phase displacement. Therefore, according to the symmetric structure of the circuit, they cancel each other out,

$$
\mathbf{V}_{A, B}=\frac{2 V_{d c}}{\pi} \cdot \cos (\Psi / 2) \pm j \frac{2 V_{d c}}{\pi} \cdot \sin (\Psi / 2)
$$




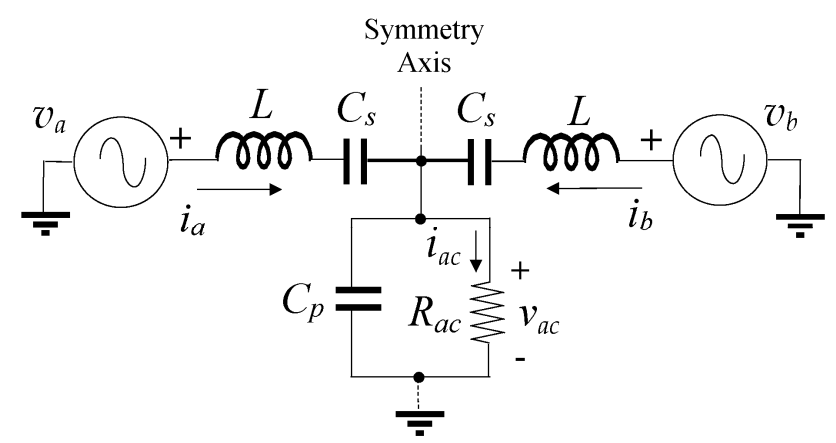

Figure 2. Simplified circuit for steady-state analysis purposes.

The resonant inverter is reduced to the equivalent circuit shown in Figure 3, where the input voltage, $\mathbf{V}_{A B}$, is the sum of the real part of $\mathbf{V}_{A}$ and $\mathbf{V}_{B}$, i.e., the common-mode voltage.

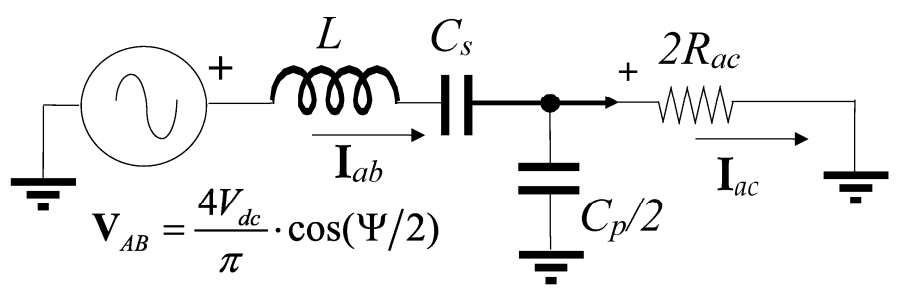

Figure 3. Two-phase $L C_{s} C_{p}$ resonant inverter. Circuit simplified according to the Bisection Theorem.

The parallel parameters of the $L C_{s} C_{p}$ inverter are summarized in Table 1.

Table 1. Parallel parameters of the $L C_{s} C_{p}$ resonant inverter.

\begin{tabular}{ccc}
\hline Parallel Resonant Frequency & Parallel Characteristic Impedance & Parallel Quality Factor \\
\hline$\omega_{p}=\frac{1}{\sqrt{L C_{p} / 2}}$ & $Z_{p}=\omega_{p} L=\frac{2}{\omega_{p} C_{p}}$ & $Q_{p}=\frac{2 R_{a c}}{Z_{p}}$ \\
\hline
\end{tabular}

From the circuit in Figure 3, the phasor of the output current on the AC side of the converter is obtained,

$$
\mathbf{I}_{a c}=\frac{4 V_{d c} \cos (\Psi / 2)}{\pi Z_{p}} \frac{1}{Q_{p}\left[1-\left(\frac{\omega}{\omega_{p}}\right)^{2}+\frac{C_{p}}{2 C_{s}}\right]-j\left(\frac{\omega_{p}}{\omega} \cdot \frac{C_{p}}{2 C_{s}}-\frac{\omega}{\omega_{p}}\right)}
$$

Since LEDs are current-driven devices, current source behavior of the driver circuit is highly desirable. For the $L C_{s} C_{p}$ resonant inverter, the current source behavior is achieved by fixing the switching frequency at $\omega=\Omega_{0}$,

$$
\Omega_{0}=\omega_{p} \sqrt{1+C_{p} / 2 C_{s}}
$$

Working at $\Omega_{0}$, it is possible to achieve the zero voltage switching (ZVS) mode of the transistors of the converter, minimizing the switching loss. Upon substitution of (4) into (3), it can be verified that the phasor of the output current on the AC side has no dependence on the load, while it is modulated with $\Psi$.

$$
\mathbf{I}_{a c}=-j \frac{4 V_{d c} \sqrt{1+C_{p} / 2 C_{s}}}{\pi Z_{p}} \cos (\Psi / 2)
$$

\subsection{Center Tap Rectifier}

Next, $R_{a c}$ for the model and the LED current, $I_{0}$, are calculated. The center tap rectifier with an $L C$ filter at the output is shown in Figure 4, where $r_{0}$ includes both the dynamic resistance of the LED lamp, 
$r_{d}$, and the shunt resistor, $R_{s}$, so that, $r_{o}=r_{d}+R_{s}$. The rectifier stage removes the high-frequency ripple generated by the operation of the inverter. Under this consideration, the low ripple approximation is used [9]. Thus, the rectifier section is modeled at low frequency by its averaged variables.

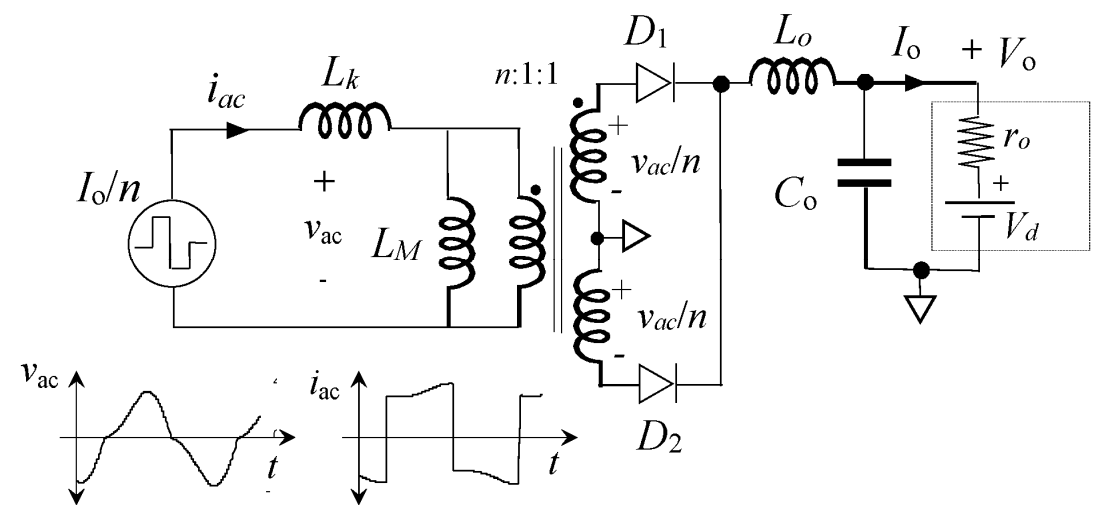

Figure 4. Center tap rectifier.

Taking into account that the rectifier and filter impose a square waveform for the current on the primary side of the transformer [9], the first harmonic of the square waveform is used to obtain the relationship between the amplitude of the AC side current, $\hat{I}_{a c}$, and the DC side current, $I_{0}$,

$$
I_{o}=\frac{n \pi}{4} \cdot \hat{I}_{a c}
$$

On the other hand, the output voltage, $V_{o}$, is the voltage across the LED lamp and the shunt resistor $R_{s}$. Carrying out the power balance between the AC and DC sides of the converter, $V_{o}$ is obtained,

$$
V_{o}=\frac{2 \hat{V}_{a c}}{n \pi}=r_{o} \cdot I_{o}+V_{d}
$$

From (7) and (8), the large-signal lamp model is represented on the AC side by,

$$
\hat{V}_{a c}=\frac{n^{2} \pi^{2}}{8} r_{0} \cdot \hat{I}_{a c}+\frac{n \pi}{2} \cdot V_{d}
$$

From (9), the impedance seen from the AC side in steady-state, $R_{a c}$, is:

$$
R_{a c}=\frac{\pi^{2}}{8} n^{2}\left(r_{o}+\frac{V_{d}}{I_{o}}\right)=\frac{\pi^{2}}{8} n^{2} R_{o}
$$

Since the LED lamp is connected on the DC side, working with (5) and (6), the DC output current is obtained as a function of the inverter parameters:

$$
\mathrm{I}_{o}=\frac{2 n V_{d c} \sqrt{1+C_{p} / 2 C_{s}}}{Z_{p}} \cos (\Psi / 2)
$$

From (10), (5) and Figure 1, it can be observed that the proposed converter enables control of the LED lamp current by adjusting the phase displacement angle, $\Psi$, between the midpoint voltages $v_{a}$ and $v_{b}$. In this approach, the switching frequency of the converter is constant at $\Omega_{0}$, which simplifies the control circuit and optimizes the magnetic components' design. 


\section{Envelope Model of the Resonant Inverter Stage}

Low-frequency perturbations cause the modulation of voltage and current waveforms in the resonant circuit [10]. Assuming the FHA, the general form of any modulated waveform is given in (11),

$$
x(t)=A(t) \cos [\omega t+\phi(t)]
$$

Given that the control of the converter is performed at constant switching frequency, the variation of the control parameter $\Psi(\mathrm{t})$ results in the amplitude modulation of all the variables [10],

$$
x(t)=\operatorname{Re}\left[A(t) \cdot e^{j \omega t}\right]
$$

Using (12), the time-varying amplitude of the current and voltage phasors are defined with (13) and (14), assuming constant switching frequency, $\omega=\Omega_{0}$,

$$
\begin{aligned}
i(t) & =\operatorname{Re}\left[I(t) \cdot e^{j \Omega_{0} t}\right] \\
v(t) & =\operatorname{Re}\left[V(t) \cdot e^{j \Omega_{0} t}\right]
\end{aligned}
$$

Upon substitution of (13) and (14) into the equations of the inductor voltage and capacitor current, the envelope model of the resonant circuit components is obtained [8].

$$
\begin{gathered}
L \frac{d I(t)}{d t}+j \Omega_{0} L I(t)=V(t) \\
C \frac{d V(t)}{d t}+j \Omega_{0} C V(t)=I(t)
\end{gathered}
$$

From (15) and (16), it can be seen that the envelope model of the reactive components incorporates an imaginary resistor [11]. The imaginary resistor represents the steady-state impedance of the corresponding reactive element at the switching frequency.

The input voltage to the resonant circuit is obtained considering that the control angle, $\Psi(t)$, is time-dependent, and it modulates the amplitude of the input voltage.

$$
v_{a b}(t)=\operatorname{Re}\left[\frac{4 V_{d c}}{\pi} \cos (\Psi(t) / 2) \cdot e^{j \Omega_{0} t}\right]
$$

Therefore, the voltage source applied to the envelope model is given by,

$$
\bar{V}_{a b}(t)=\frac{4 V_{d c}}{\pi} \cos (\Psi(t) / 2)
$$

Finally, applying (15), (16) and (18) to the circuit of Figure 3, the envelope model of the $L C_{s} C_{p}$ resonant inverter stage is obtained,

Observing Figure 5, it is possible to reduce the order of the model by assuming that the switching frequency $\Omega_{o}$ is much higher than the frequency of any perturbation. In this way, $L$ and $C_{S}$ along with the associated imaginary resistances can be lumped in an equivalent inductor in series connection with the steady-state $C_{s}$ impedance as shown next,

$$
\frac{1}{\left(s+j \Omega_{0}\right) C_{s}} \approx \frac{s}{\Omega_{0}^{2} C_{s}}+\frac{1}{j \Omega_{0} C_{s}}=s L\left(\frac{\omega_{s}}{\Omega_{0}}\right)^{2}+\frac{1}{j \Omega_{0} C_{s}}
$$

where, $\omega_{S}$ is the resonant frequency of the series branch $L-C_{S}$.

$$
\omega_{s}=\frac{1}{\sqrt{L C_{s}}}=\frac{\Omega_{o}}{\sqrt{1+2 C_{s} / C_{p}}}
$$




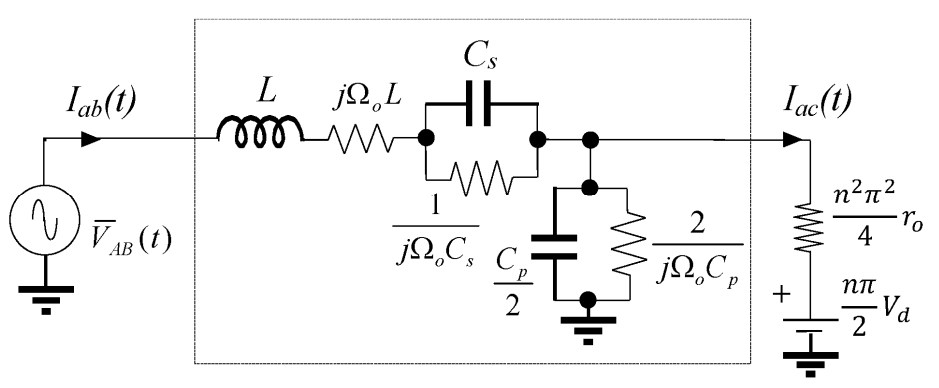

Figure 5. Large-signal envelope model of the inverter stage.

Then, after grouping terms, the envelope model of the series branch is further reduced to the equivalent inductance, $L_{r d}$, in series with an imaginary resistor, $j X_{r d}$, defined as follows:

$$
\begin{gathered}
L_{r d}=L\left(\frac{1+C_{p} / C_{s}}{1+C_{p} / 2 C_{s}}\right) \\
X_{r d}=\frac{\Omega_{o} L}{1+C_{p} / 2 C_{s}}
\end{gathered}
$$

In this way, the model shown in Figure 5 is simplified, resulting in the one in Figure 6.

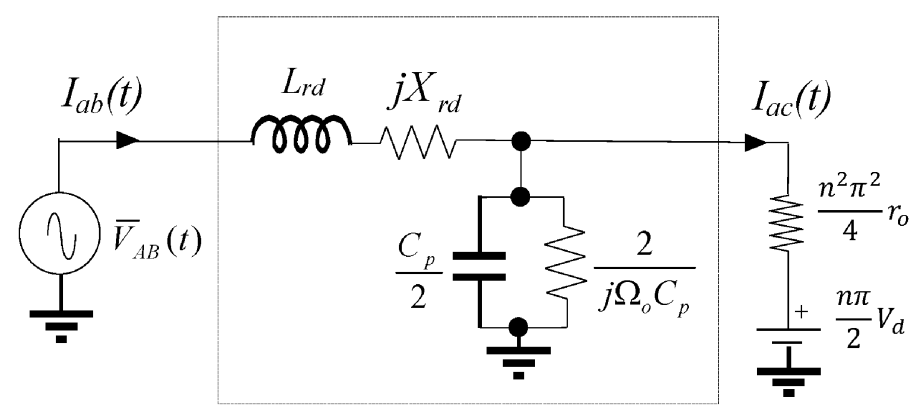

Figure 6. Large-signal reduced-order envelope model of the inverter stage.

It should be noted that the reduced-order model incorporates the main dynamic effect of the capacitance $C_{s}$ at low frequency, which is expressed by the new inductance $L_{r d}$.

\subsection{Real and Imaginary Subcircuits in the Model}

According to the procedure explained in [11], the complex circuit in Figures 5 and 6 can be simulated with Spice if the circuit is split into its real and imaginary parts. The corresponding real and imaginary subcircuits are shown in Figures 7 and 8 respectively. Voltages and currents are obtained by composing the ones in the real and imaginary circuit, e.g., $I_{a c}$. This method is also used for the analysis.

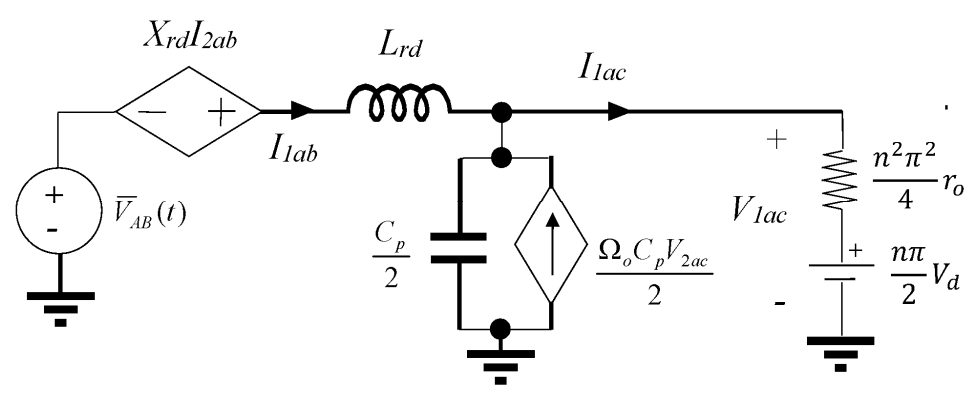

Figure 7. Real part of the large-signal reduced-order envelope model of the inverter stage. 


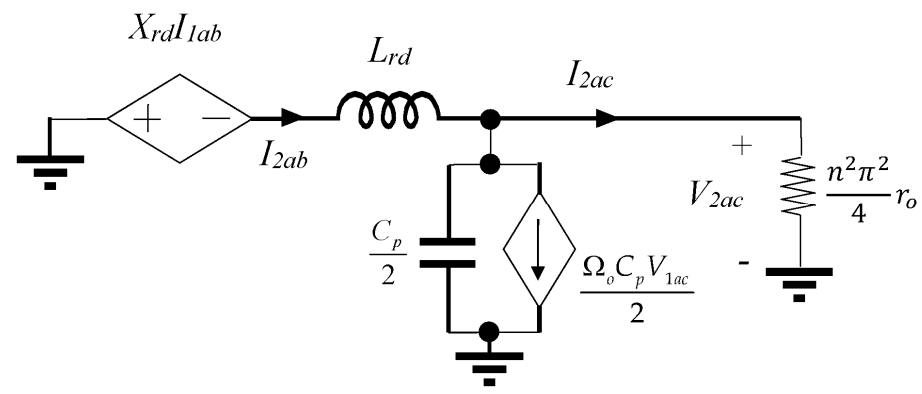

Figure 8. Imaginary part of the large-signal reduced-order envelope model of the inverter stage.

The envelope of the output current on the AC side is calculated composing its orthogonal components.

$$
\bar{I}_{a c}=\left|I_{a c}\right|=\sqrt{I_{1 a c}^{2}+I_{2 a c}^{2}}
$$

Once the envelope of the output current on the AC side is obtained, the calculation of the DC output current is straightforward applying (6) as represented in Figure 9.

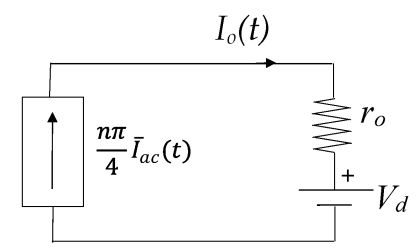

Figure 9. DC output current obtained from the envelope of the AC side output current.

\subsection{Small-Signal Envelope Model}

Perturbing and linearizing (23), the small-signal component of the AC side load current envelope is obtained,

$$
\hat{\bar{i}}_{a c}=\frac{I_{1 a c}}{\bar{I}_{a c}} \cdot \hat{i}_{1 a c}+\frac{I_{2 a c}}{\bar{I}_{a c}} \cdot \hat{i}_{2 a c}
$$

In (24), $\hat{\imath}_{1 a c}$ and $\hat{\imath}_{2 a c}$ are the small-signal components of $I_{1 a c}$ and $I_{2 a c}$. Solving the circuits in Figures 7 and 8 , the steady-state components of the output current, $I_{1 a c}$ and $I_{2 a c}$, are obtained. From (5), at frequency $\Omega_{0}$ where the inverter behaves as a current source, the phasor of the steady-state output current is purely imaginary, i.e., $I_{1 a c}=0$ and, therefore, $I_{a c}=I_{2 a c}$, so that,

$$
\hat{\bar{i}}_{a c}=-\hat{i}_{2 a c}
$$

The small-signal current obtained in (25) is applied to the input impedance of the loaded output filter, $z_{i f}$, as shown in Figure 10.

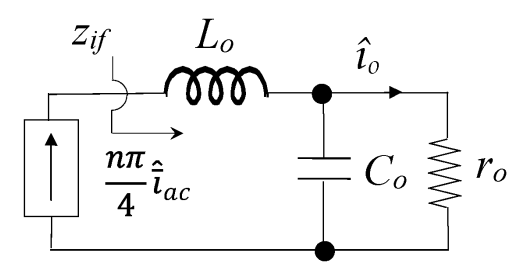

Figure 10. Small-signal response of the output current. 
In order to obtain the linear small-signal plant of the resonant inverter, the modulation signal, $\Psi$, has to be perturbed [11], which results in perturbation of the input voltage. Then, the small-signal perturbation, $\phi$, is added to $\Psi$ :

$$
\Psi(t)=\Psi_{o}+\phi
$$

Substituting (26) into (17) and extracting the small-signal component,

$$
\hat{v}_{a b}=-\phi \cdot \frac{2 V_{d c}}{\pi} \cdot \sin \left(\frac{\Psi_{o}}{2}\right)
$$

The AC side small-signal load impedance is the input impedance of the output filter reflected on the AC side,

$$
z_{a c}=\frac{n^{2} \pi^{2}}{4} z_{i f}
$$

The resulting small-signal reduced-order model of the phase-controlled $L C_{s} C_{p}$ resonant converter is shown in Figure 11.

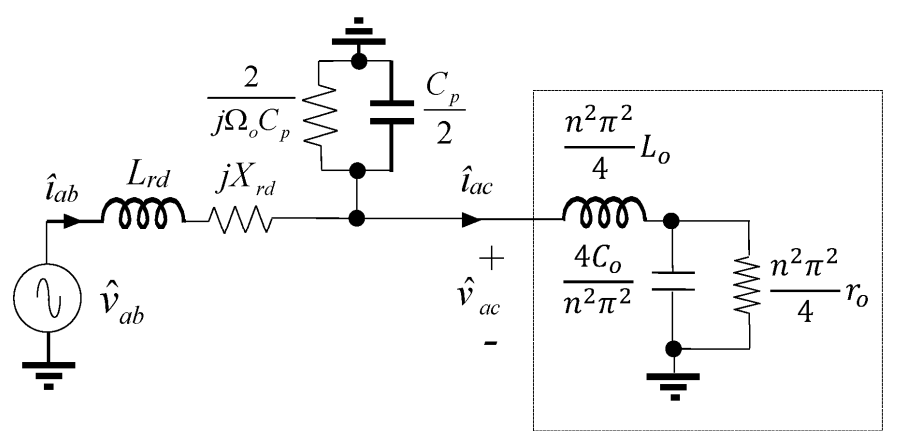

Figure 11. Small signal of the reduced-order envelope model of the AC side.

Splitting the model of Figure 11 into real and imaginary sub-circuits, the small-signal components $\hat{\imath}_{1 a c}$ and $\hat{\imath}_{2 a c}$ are calculated. Upon substitution of $\hat{\imath}_{2 a c}$ into (25), the control-to-output current transfer function in the AC side is obtained.

\section{Experimental Results}

The experimental prototype and lamp setup are shown in Figure 12.

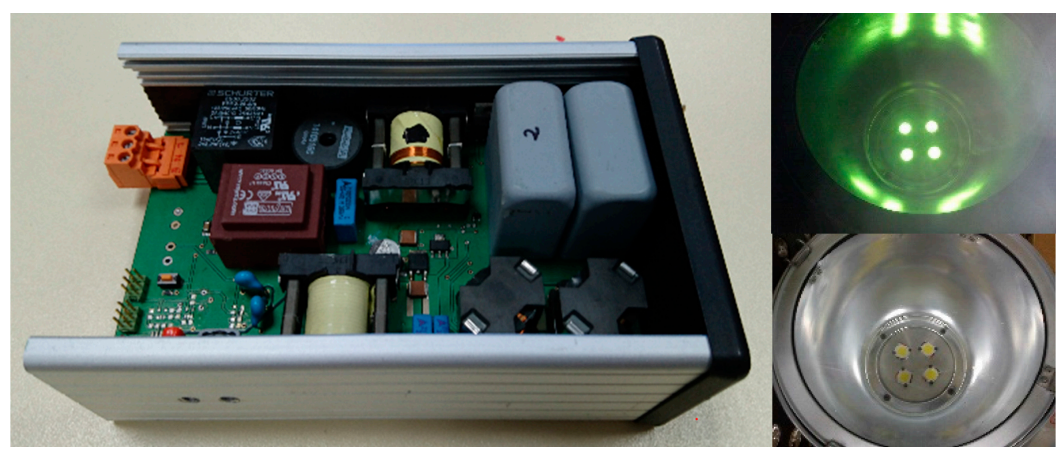

Figure 12. Experimental prototype and lamp composed of four LED arrays BXRA-C2000. Case size is $16 \mathrm{~cm} \times 10 \mathrm{~cm} \times 5 \mathrm{~cm}$.

The lamp consists of four BXRA-C2000 LED arrays in series connection with a nominal current $I_{o}=1.75 \mathrm{~A}$, output power $P_{o}=120 \mathrm{~W}$, output voltage $V_{o}=68.6 \mathrm{~V}$ and equivalent DC load, and $R_{o}=39.2 \Omega$. The control angle under nominal conditions is $\Psi_{o}=45^{\circ}$, which provides full control 
capability. The input DC voltage is $V_{d c}=400 \mathrm{~V}$. The transformer's turns ratio is chosen to be $n=2$, which implies that $R_{a c}=193.4 \Omega$. The capacitor ratio is $C_{p} / C_{s}=0.1$.

After collecting all the input data, the step-by-step design sequence of the converter is as follows: Upon substitution of $n, V_{d c}, \Psi_{o}$ and $I_{o}$ into (10), the characteristic impedance is obtained, $Z_{p}=433 \Omega$. From Table 1 , the parallel quality factor is $Q_{p}=0.894$. The resulting value of the quality factor produces $48^{\circ}$ phase lag of the current referred to the voltage in the transistors, which is enough for ensuring the ZVS mode under all operating conditions. The switching frequency is set at $\Omega_{\mathrm{o}}=2 \pi(100 \mathrm{kHz})$. From (4), the parallel resonant frequency is $\omega_{p}=2 \pi(97.6 \mathrm{kHz})$. Finally, the reactive components are $L=705 \mu \mathrm{H}, C_{p}=7.5 \mathrm{nF}$ and $C_{s}=75 \mathrm{nF}$. The output filter components are $L_{o}=150 \mu \mathrm{H}$ and $C_{o}=3.3 \mu \mathrm{F}$. The use of electrolytic capacitors, even in the power factor correction stage, is avoided in order to achieve high circuit reliability [12]. The resonant inverter stage was implemented using IRF840LC MOSFET transistors. The resonant inductors were built using the ferrite core RM10 of material 3C90. The experimental lamp current and voltage under nominal conditions are shown in Figure 13, where $\mathrm{PhA}$ and $\mathrm{PhB}$ are the signals for driving phases $\mathrm{A}$ and $\mathrm{B}$ of the converter.

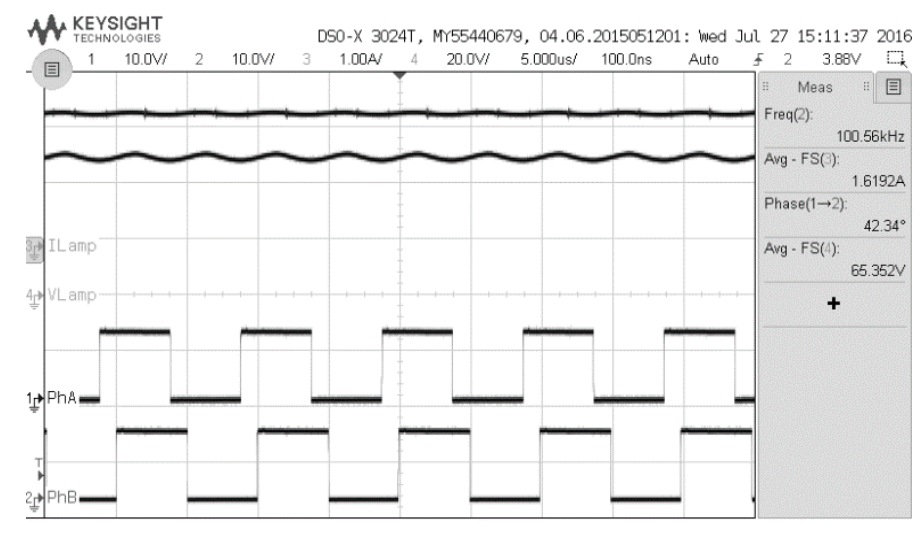

Figure 13. Lamp current, lamp voltage and control signals in steady state.

From Figure 13, the driver performance under nominal conditions can be verified. The current of the LED lamp is $I_{o}=1.62 \mathrm{~A}$. The phase displacement angle, $\Psi$, between the drive signals $\mathrm{PhA}$ and $\mathrm{PhB}$ is $\Psi=42.3^{\circ}$. The efficiency of the proposed driver is $92 \%$.

\subsection{Validation of the Converter Dynamic Model}

The core of the control circuit is a phase modulator [13], which is implemented using a sawtooth carrier (leading edge) signal, a comparator and two monostable multivibrators. The control voltage, $v_{\mathcal{C}}$, and the carrier are compared, generating a PWM signal that triggers the drive signals of phases $\mathrm{A}$ and $\mathrm{B}$ of the converter. The waveforms are shown in Figure 14.

The LT-Spice description of the real and imaginary sub-circuits of the small-signal model are shown in Figure 15. The dynamic resistance of the LED lamp is $r_{d}=5 \Omega$. The shunt resistor is $R_{s}$ $=0.5 \Omega$, so the dynamic output resistance is $r_{o}=5.5 \Omega$. The ESR of the output filter components is considered. The input voltage for the AC simulation of the model is defined by (27), taking into account that the gain of the phase modulator is $G_{\phi}=-0.7 \mathrm{rad} / \mathrm{V}$, and the amplitude of the perturbation is $v_{c}=500 \mathrm{mV}$.

The open-loop control-to-output current AC characterization, shown in Figure 16, has been carried out injecting a constant amplitude perturbation signal, $v_{\mathcal{C}}=0.5 \mathrm{~V}$, between $51.2 \mathrm{~Hz}$ and $51.2 \mathrm{kHz}$, using an Agilent 35670A Dynamic Signal Analyzer. From Figure 16, it can be observed that the proposed model and the experimental results are in good agreement up to $30 \mathrm{kHz}$, verifying a first-order dominant behavior of the resonant converter. 


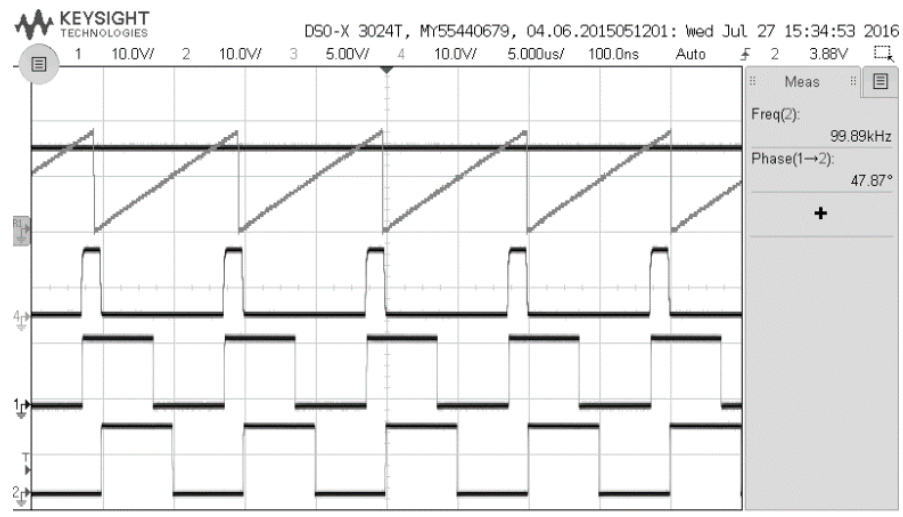

Figure 14. Waveforms of the phase modulator for $\Psi=45^{\circ}$. From top to bottom: Sawtooth carrier signal. Control signal, $v_{\mathcal{C}}$. Pulsewidth modulated signal. Drive signals applied to the resonant converter branches.
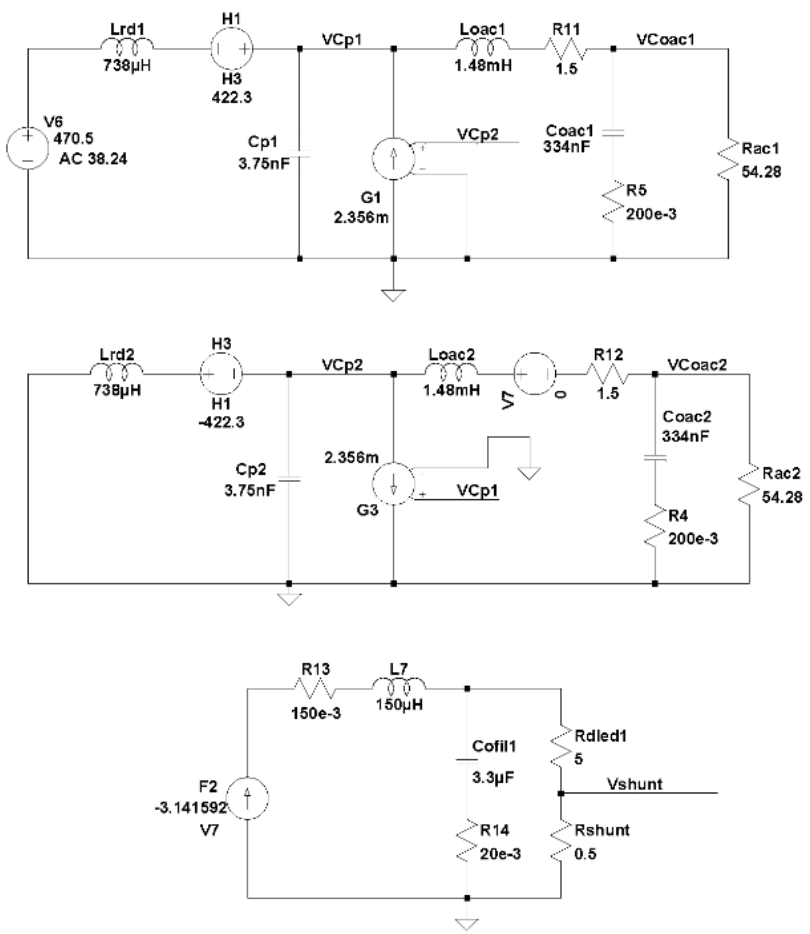

Figure 15. LT-Spice implementation of the small-signal model of the resonant converter.

The experimental bandwidth $\omega_{H}=2 \pi(9 \mathrm{kHz})$ is wide enough to implement the PWM dimming at a frequency $f_{P W M}=2 \mathrm{kHz}$, which exceeds the recommendation regarding the minimum frequency for avoiding flicker perception given by the IEEE PAR 1789. The result is in good agreement with (29).

$$
\omega_{H}=\frac{1}{r_{o} C_{o}}
$$

The reflected impedance of the output filter on the AC side produces a peak of resonance, whose position is given by,

$$
\omega_{f}=\frac{2}{n \pi \sqrt{L_{o} C_{p}}}
$$

In order to preserve the first-order behavior of the converter and achieve wide bandwidth, the converter should be designed to fulfill $\omega_{H}<\omega_{f}$. 

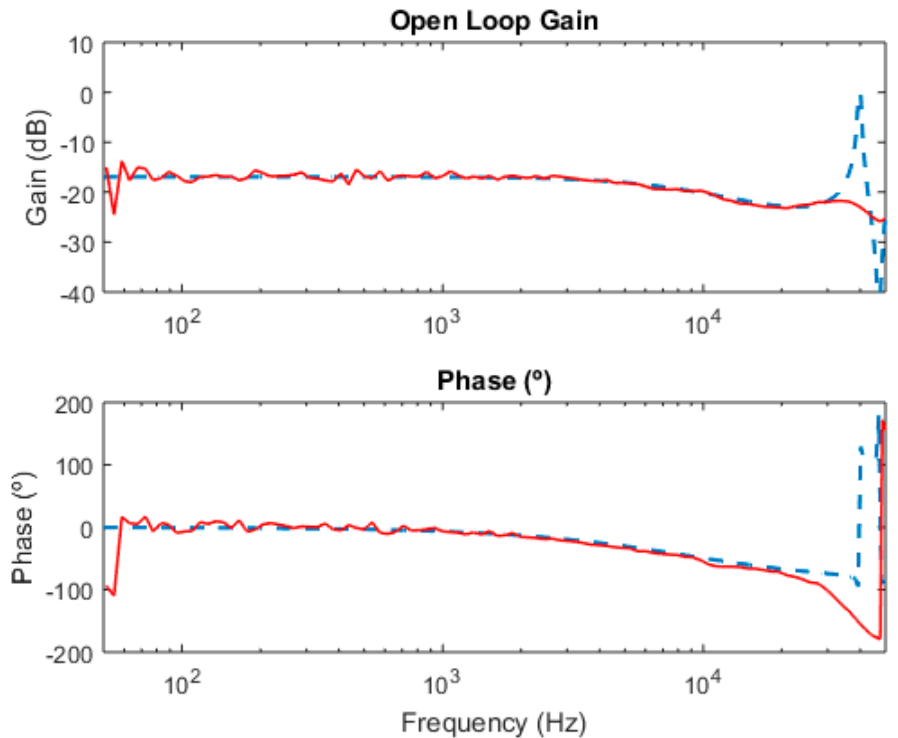

Figure 16. Experimental (solid red line) and predicted by the proposed model (dashed blue line) AC response of the converter.

\subsection{PWM Control of the Lamp Current}

As stated previously, the converter enables the implementation of the PWM control of the lamp current. The off-time is achieved by imposing $\Psi=180^{\circ}$. A good feature of the phase-shifted mode PWM is that the resonant tank is not fully discharged, which prevents uncontrolled on/off transient oscillations of the converter variables. The experimental waveform for $50 \%$ dimmed operation is shown in Figure 17. The current during the on-time is the nominal one of the LED lamp, $I_{o}=1.7 \mathrm{~A}$, which assures the quality of the light, without any chromatic modification. The average current of the lamp is $0.86 \mathrm{~A}$, and the power is $59 \mathrm{~W}$, verifying the operation at $50 \%$ of the nominal power. The efficiency of the proposed driver, measured at $50 \%$ of the nominal current, is $87 \%$.

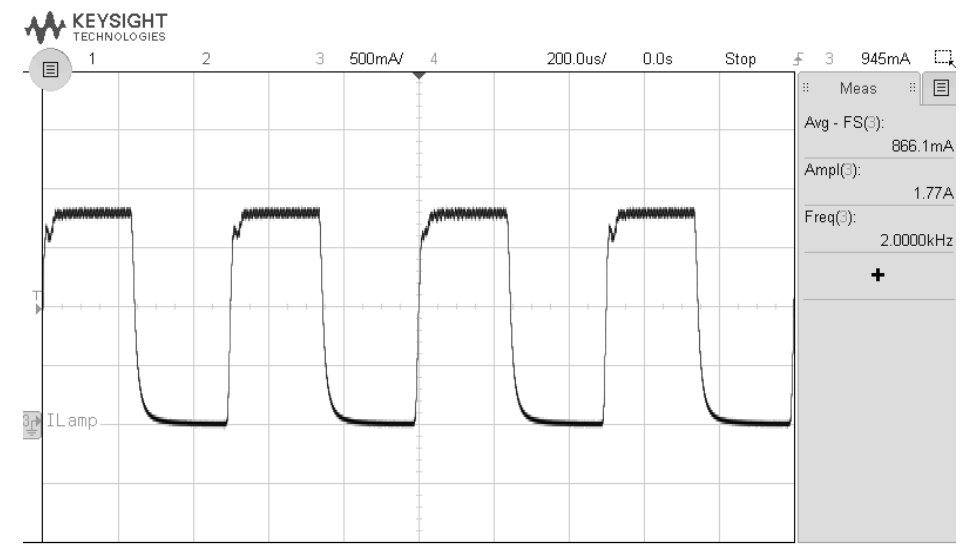

Figure 17. Experimental current of the lamp at 50\% dimmed operation achieved by the phase-shifted PWM.

The converter bandwidth, close to $10 \mathrm{kHz}$, also enables data transmission, as is shown in Figure 18. The bit rate depends on the bit codification. For example, a light-based Amplitude-Shift Keying ASK modulation with an on-off keying codification can be implemented by switching the lamp current between two levels, as is shown in Figure 18. This on-off keying codification achieves a bit rate that is twice the frequency of the signal as two bits are transmitted during a cycle of the signal. In order to achieve the minimum degradation of the light quality during data transmission, the level of " 1 " and 
" 0 " were fixed at $1.7 \mathrm{~A}$ and $1 \mathrm{~A}$, respectively. At these levels, the average current of the lamp is $1.3 \mathrm{~A}$ and the power is $92 \mathrm{~W}$, close to the nominal conditions. During data transmission, the efficiency of the driver is approximately equal to the value under nominal conditions.

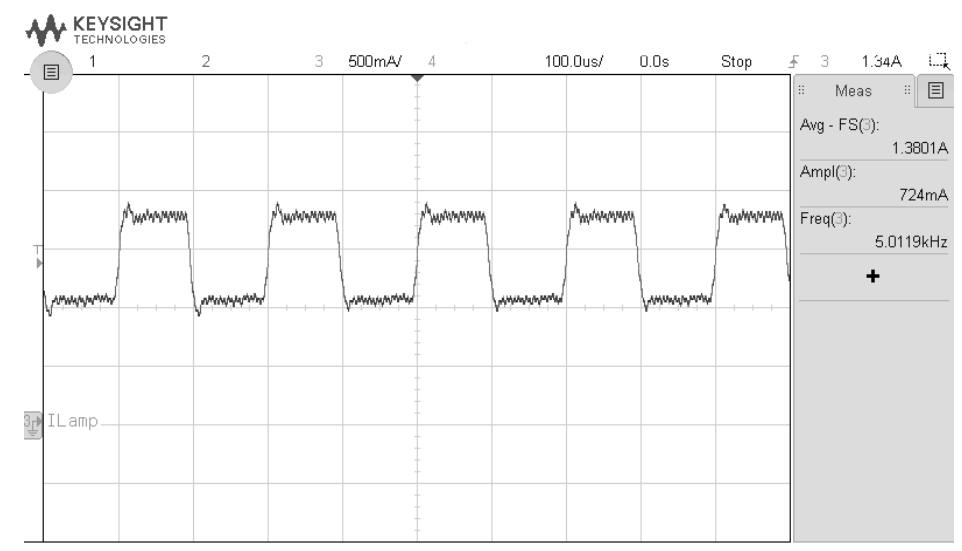

Figure 18. Bi-level modulation of the lamp current for implementing data transmission at $10 \mathrm{kB} / \mathrm{s}$.

\section{Discussion}

The small-signal model obtained from the reduced envelope model of the two-phase phase-shift-modulated $L C_{s} C_{p}$ resonant converter, proposed as a driver for high-power LED lamps, has been validated with simulation and experimentally up to tens of $\mathrm{kHz}$ frequency. The output filter after the rectifier stage imposes on the converter control-to-output dominant single pole, so that a simple adjustment of the output capacitance in consistency with the load is enough to meet the IEEE PAR 1789 recommendations to perform wide-range PWM dimming, preventing any flicker perception, and to implement a VLC protocol using multilevel modulation. Experimental results show a ASK modulation capability up to $10 \mathrm{kB} / \mathrm{s}$. Since no excessive variation of the resonant tank energy occurs during the phase-shift modulation transients, sharp amplitude modulation transients with no significant over-shoots or oscillations are achieved.

Author Contributions: Conceptualization, C.B. and F.J.A.; methodology, C.B.; validation, C.B., R.C. and F.J.D.; formal analysis, C.B.; investigation, C.B. and F.J.A.; writing-original draft preparation, C.B.; writing-review and editing, R.C. and F.J.D.; visualization, C.B.; supervision, F.J.A.; project administration, C.B. and F.J.A.; funding acquisition, F.J.A. All authors have read and agreed to the published version of the manuscript.

Funding: This work has been sponsored by the Spanish Ministry of Science and Innovation and the EU through the projects CICYT-FEDER-TEC2014-52316-R: "Estimation and Optimal Control for Energy Conversion with Digital Devices, ECOTRENDD" and RTI2018-095138-B-C31: "Power Electronics for the Grid and Industry Applications".

Conflicts of Interest: The authors declare no conflict of interest.

\section{References}

1. Crawford, M. LEDs for Solid-State Lighting: Performance Challenges and Recent Advances. IEEE J. Sel. Top. Quantum Electron. 2009, 15, 1028-1040. [CrossRef]

2. Loo, K.H.; Lai, Y.M.; Tan, S.-C.; Tse, C.K. On the Color Stability of Phosphor-converted White LEDs Under DC, PWM and Bilevel Drive. IEEE Trans. Power Electron. 2012, 27, 974-984. [CrossRef]

3. Wilkins, A.; Veitch, J.; Lehman, B. LED lighting flicker and potential health concerns: IEEE standard PAR1789 update. In Proceedings of the 2010 IEEE Energy Conversion Congress and Exposition, Atlanta, GA, USA, 12-16 September 2010; pp. 171-178.

4. Carli, R.; Dotoli, M.; Pellegrino, R. A decision-making tool for energy efficiency optimization of street lighting. Comput. Oper. Res. 2018, 96, 223-235. [CrossRef]

5. Pandharipande, A.; Caicedo, D. Smart indoor lighting systems with luminaire-based sensing: A review of lighting control approaches. Energy Build. 2015, 104, 369-377. [CrossRef] 
6. O’Brien, D.C.; Zeng, L.; Le-Minh, H.; Faulkner, G.; Walewski, J.W.; Randel, S. Visible light communications: Challenges and possibilities. In Proceedings of the 2008 IEEE 19th International Symposium on Personal, Indoor and Mobile Radio Communications, Cannes, France, 15-18 September 2008.

7. Zheng, S.; Czarkowski, D. Dynamics of a phase-controlled series-parallel resonant converter. In Proceedings of the 2002 IEEE International Symposium on Circuits and Systems. Proceedings (Cat. No.02CH37353), Phoenix-Scottsdale, AZ, USA, 26-29 May 2002; pp. 819-822.

8. Czarkowski, D.; Kazimierczuk, M. Phase-controlled series-parallel resonant converter. IEEE Trans. Power Electron. 1993, 8, 309-319. [CrossRef]

9. Steigerwald, R. A comparison of half-bridge resonant converter topologies. IEEE Trans. Power Electron. 1988, 3, 174-182. [CrossRef]

10. Yin, Y.; Zane, R.; Glaser, J.; Erickson, R. Small-signal analysis of frequency-controlled electronic ballasts. IEEE Trans. Circuits Syst. I Regul. Pap. 2003, 50, 1103-1110. [CrossRef]

11. Lineykin, S.; Ben-Yaakov, S. Unified SPICE compatible model for large and small-signal envelope simulation of linear circuits excited by modulated signals. IEEE Trans. Ind. Electron. 2006, 53, 745-751. [CrossRef]

12. Pervaiz, S.; Kumar, A.; Afridi, K.K. A compact electrolytic-free two-stage universal input offline LED driver. In Proceedings of the 2016 IEEE Applied Power Electronics Conference and Exposition (APEC), Long Beach, CA, USA, 20-24 March 2016; pp. 2395-2400.

13. Talarico, C.; D'Amato, G.; Coviello, G.; Avitabile, G. A high precision phase control unit for DDS-based PLLs for 2.4-GHz ISM band applications. In Proceedings of the 2015 IEEE 58th International Midwest Symposium on Circuits and Systems (MWSCAS), Fort Collins, CO, USA, 2-5 August 2015. [CrossRef]

(C) 2019 by the authors. Licensee MDPI, Basel, Switzerland. This article is an open access article distributed under the terms and conditions of the Creative Commons Attribution (CC BY) license (http://creativecommons.org/licenses/by/4.0/). 